Case Report

\title{
Laparoscopic Tissue Repair of an Acutely Incarcerated Spigelian Hernia
}

\author{
Yagan Pillay a,*, Rufaro Asefa ${ }^{b}$ \\ ${ }^{a}$ Department of Surgery, University of Saskatchewan, Victoria Hospital, Prince Albert, Saskatchewan, Canada \\ ${ }^{b}$ St. George's University, University Centre, Grenada, West Indies, Canada
}

Article history:

Received: July 15, 2020

Revised: October 24, 2020

Accepted: October 25, 2020

${ }^{*}$ Corresponding Author:

Yagan Pillay

Department of Surgery, University of

Saskatchewan, Victoria Hospital, Prince

Albert, Saskatchewan, Canada

Email: yagan2pillay@yahoo.ca

\section{ORCID}

Yagan Pillay

https://orcid.org/0000-0003-1254-3179

Rufaro Asefa

https://orcid.org/0000-0001-5224-526X

\begin{abstract}
Spigelian hernias are a rare type of abdominal wall hernia. A weakness in the spigelian fascia causes abdominal contents to herniate between the abdominal muscle layers as an interparietal hernia. We present the case of a 72-year old woman with acute onset abdominal pain and distention. A computed tomography scan revealed an incarcerated spigelian hernia with small bowel obstruction. This necessitated an acute surgical intervention with a laparoscopic tissue repair. The case is unique as our patient had a large hernial neck measuring 4.8 centimeters, one of the largest in published literature. The laparoscopic tissue repair is outside the current norm for this type of hernial repair and remains a rare type of surgical herniorrhaphy. Laparoscopic ventral hernia repair in the acute setting should form part of the surgical skill set of acute care surgeons as borne out by the published literature and this case report.
\end{abstract}

Keywords: abdominal wall hernia, surgery, laparoscopic surgery, fascia

\section{Introduction}

Spigelian hernias are a rare subset of abdominal wall hernias that protrude through the transversus abdominus muscle [1] as an interparietal hernia. An incarcerated small bowel spigelian hernia is reported in this study. The presentation of a small bowel obstruction and inability to reduce the hernia preoperatively necessitated an acute laparoscopic surgical intervention. A typical hernial neck diameter would be $1-2 \mathrm{~cm}$ $[1,2]$. In this case the hernial neck diameter was $4.8 \mathrm{~cm}$ which would make this one of the largest documented spigelian hernias.

\section{Case Report}

\section{Medical history, diagnosis, and interventions}

A 72-year-old female was referred to acute care surgical services with abdominal distention and right lower quadrant pain. The pain was acute at onset and began a few hours prior to her admission to the emergency room. She had no previous episodes of this type of pain. Her pain did not radiate and was relieved by taking intravenous opioid analgesia. Her significant medical history included morbid obesity, hypothyroidism, hypercholesterolemia, and atherosclerotic disease. She had a previous open umbilical herniorrhaphy without mesh reinforcement.

Clinically she had a distended abdomen with tympanic bowel sounds. There was a palpable mass in her right lower quadrant which was tender on palpation. The abdominal wall mass was immobile and vague with no clinically discernible borders. She had no clinical signs of rebound tenderness or guarding.

An erect abdominal x-ray (Figure 1) showed signs of a small bowel obstruction without free air, and this was in keeping with perforation of a hollow viscus. Her subsequent preoperative computed tomography scan showed features of an incarcerated spigelian hernia with a hernial neck measurement of $4.8 \mathrm{~cm}$. A small bowel obstruction was also identified (Figures 2A and 2B). The patient's blood parameters showed leukocytosis. Her expanded chemistry profile, C-reactive protein, and lactate levels were within the normal range.

An informed consent for emergency laparoscopy and possible 
small bowel resection was obtained. During the diagnostic laparoscopy a spigelian hernia was identified and its small bowel and omental contents were reduced into the peritoneal cavity (Figures 3 and 4). There was ischemia in the incarcerated bowel that resolved with hernial reduction. Due to the risk of bacterial translocation the decision was made to perform tissue repair because biological mesh was not available. Turbid fluid present in the hernial sac reinforced the diagnosis of bacterial translocation (a recognized risk factor for mesh contamination and abdominal wall sepsis).

Intra corporeal suturing of the hernia neck was performed (Figures 5A and 5B) and the 2-0 V-loc (North Haven, California, USA) non-absorbable suture was secured to the abdominal wall with gallbladder clips (Figures 6A and 6B). The small bowel was laparoscopically examined from the terminal ileum to the duodenal-jejunal flexure for signs of perforation or necrosis. No perforation or necrosis was noted. The herniated bowel also displayed a lipoma (Figure 3B) on its anti-mesenteric side which may have contributed to the herniation by acting as a lead point $[3,4]$.

Consent was obtained from the patient to publish this article (prior to publication).

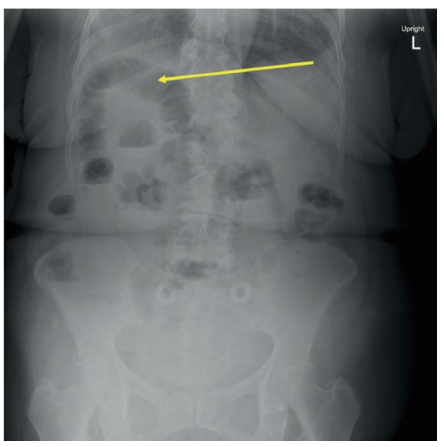

Figure 1. Small bowel distention (yellow arrow) due to spigelian hernia incarceration on an erect abdominal x-ray.

(A)

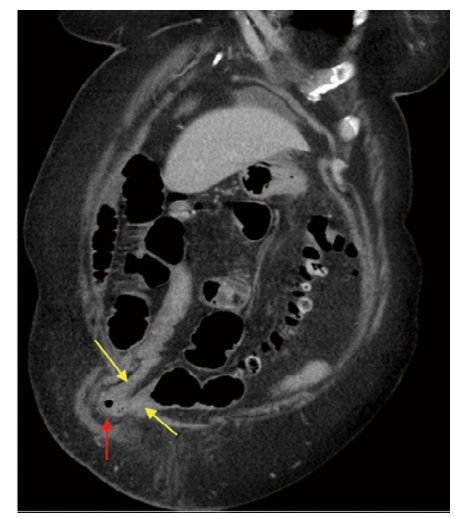

(B)

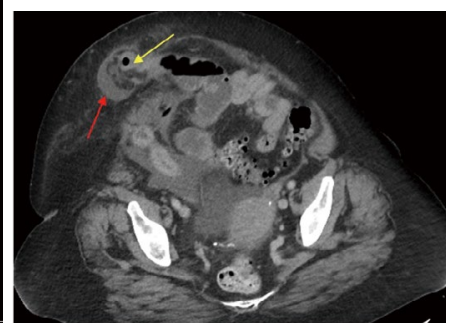

Figure 2. (A) Coronal view computed tomography scan showing the 4.8 $\mathrm{cm}$ hernia neck diameter (yellow arrows) and the small bowel content in the hernia sac (red arrow). (B) Axial view computed tomography scan showing the small bowel (yellow arrow) and turbid fluid (red arrow) in keeping with possible bacterial translocation.
(A)

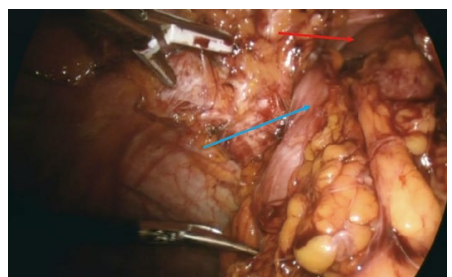

Figure 3. (A) Incarcerated small bowel (blue arrow) within the hernia neck (red arrow). (B) Incidental small bowel sub serosal lipoma (blue arrow) which may have acted as a lead point for the incarceration.

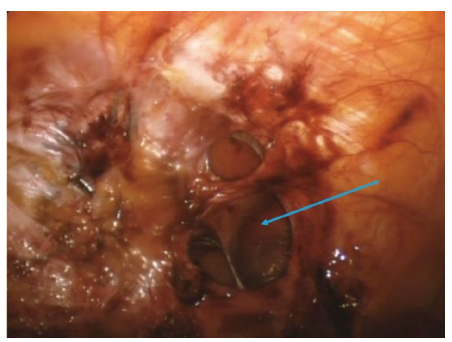

Figure 4. The exposed Swiss cheese spigelian hernia neck (blue arrow).

(A)

(B)
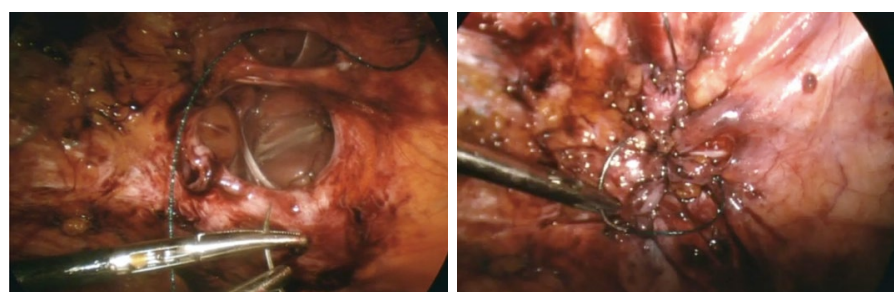

Figure 5. (A) Initiation of hernia neck closure with a V-loc nonabsorbable suture. (B) Completed closure of the hernia neck.
(A)

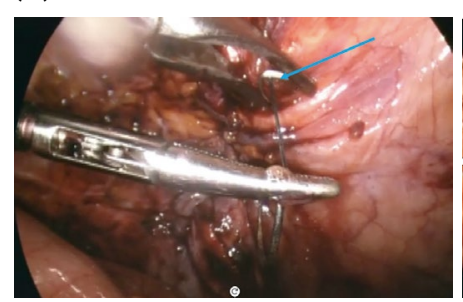

(B)

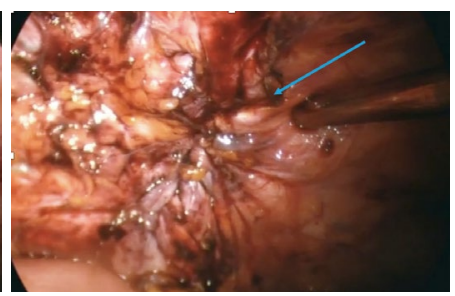

Figure 6. (A) Suture stabilization with a gallbladder clip attached to the abdominal wall (blue arrow). (B) The completed repair with the gallbladder clip in situ (blue arrow).

\section{Outcome}

The patient made an uneventful recovery and was discharged from hospital the same day. She was reviewed in ambulatory care 48 hours later and had no untoward concerns. Her abdomen was clinically soft with no palpable masses and a negative cough impulse. One-week post-surgery she remained asymptomatic and her wounds had all healed. The patient was extensively counselled about her tissue repair, the possibility of a recurrence due to the absence of a prosthetic mesh, and her morbid obesity. Surveillance of the herniorrhaphy will continue for at least 1 year. 


\section{Discussion}

Spigelian hernias are rare surgical entities with a reported incidence of $2 \%$ [1,2]. This case report is unique in the large size of the hernial neck. Laparoscopic tissue repair is a rare type of surgical herniorrhaphy due to the high recurrence rate associated with a tissue repair. The hernial neck size was quite large at $4.8 \mathrm{~cm}$ as most of the recorded diameters in published reports are between $1 \mathrm{~cm}$ and $2 \mathrm{~cm} \mathrm{[1,2].} \mathrm{This} \mathrm{is} \mathrm{the} \mathrm{largest}$ known neck diameter for a spigelian hernia. The large neck size should have decreased the risk of incarceration but this was not the case in this patient. The bowel ischemia visualized during the laparoscopy revealed the incarceration risk.

The presence of a small bowel lipoma on the anti-mesenteric surface of the incarcerated small bowel, an unusual occurrence in itself, may have lent itself to the possible etiology of incarceration despite the large hernial neck. The lipoma could have acted as a lead point for bowel incarceration.

Small bowel lipomas are atypical and are usually submucosal in location. The subserosa location of this lipoma made it a truly rare occurrence [5].

This case report demonstrated a repair method that was beyond the procedural norms for spigelian herniorrhaphy as most methods involve the use of prosthetic mesh reinforcement [6]. In this case there was a lack of access to biological mesh. In addition, due to the possible issue of bowel ischemia, and the subsequent risk of bacterial translocation into the hernial sac and anterior abdominal wall, the decision was made intraoperatively not to perform mesh repair. Moreover, the patient's comorbidities compounded this decision.

The biological mesh method would have provided prosthetic reinforcement whilst alleviating the risk of mesh contamination, however the tissue repair method was performed despite the higher risk of recurrence compared with prosthetic reinforcement of the spigelian hernia [7]. The surgeon and patient had an extensive post-operative discussion about this and the relatively higher risk for a hernial recurrence.

In conclusion, this clinical case is an example of a rarely reported spigelian hernia with the largest reported neck size which measured $4.8 \mathrm{~cm}$, which was successfully treated (with ongoing monitoring) using the tissue repair method. It is our fervent desire that this case report adds to the surgical armamentarium of acute care surgeons and that an acute repair of ventral wall hernias form part of their surgical skill set.

\section{Conflicts of Interest}

There authors have no conflicts of interest to declare.

\section{Acknowledgements}

We would like to thank our patient for giving her consent and allowing us to publish this case report.

\section{Funding}

No financial support was provided for this case study.

\section{References}

[1] Mederos R, Lamas JR, Alvarado J, Matos M, Padron I, Ramos A. Laparoscopic diagnosis, and repair of Spigelian hernia: A case report and literature review. Int J Surg Case Rep 2017;31:184-7.

[2] Griffin AS, Schaefer NR, Jeyarajan E, Cross T. Concurrent Spigelian hernia and falciform ligament hernia in a 67-year-old female. Int J Surg Case Rep 2015;13:27-9.

[3] Lavin A, Gupta A, Lopez-Viego M, Buicko JL. Incarcerated Spigelian Hernias: A Rare Cause of a High-grade Small Bowel Obstruction. Cureus 2020;12(3):e7397.

[4] Konik RD, Rhodes RA. Complete small bowel obstruction without intussusception due to a submucosal lipoma. J Surg Case Rep 2018;2018(7):rjy155.

[5] Pagaro P, Deshpande A. Lipoma of small intestine. Med J Dr DY Patil Univ 2015;8(4):525-7.

[6] Velimezis G, Vassos N, Kapogiannatos G, Koronakis D, Salpiggidis C, Perrakis E, et al. Strangulation and necrosis of right hemicolon as an extremely rare complication of Spigelian hernia. Arch Med Sci 2016;12(2):469-72.

[7] Birindelli A, Sartelli M, Di Saverio S, Coccolini F, Ansaloni L, van Ramshorst GH, et al. 2017 update of the WSES guidelines for emergency repair of complicated abdominal wall hernias. World J Emerg Surg 2017;12:37. 